\title{
The flux-gap between bright and dark solar magnetic structures
}

\author{
K. G. Puschmann and E. Wiehr
}

Institut für Astrophysik, Friedrich-Hund-Platz 1, 37077 Göttingen, Germany

e-mail: [kgp; ewiehr]@astro.physik.uni-goettingen.de

Received 21 March 2005 / Accepted 1 September 2005

\section{ABSTRACT}

The upper size limit of solar small-scale magnetic flux concentrations ("G-band bright points", BP) is reconsidered from speckle-reconstructed images taken at the 1-m SST on La Palma. The size-histogram shows a sharp drop towards $250 \mathrm{~km}$ diameter, variation of the noise filter threshold diminishes that value due to segmentation of the elongated structures. A further artificial segmentation of still elongated (i.e. not round) $\mathrm{BP}$ indicates that the upper limit may well be below $200 \mathrm{~km}$ diameter, corresponding to a flux smaller than $2.5 \times 10^{17} \mathrm{Mx}$ which is more than 40 times smaller than that of smallest dark (mini-) pores. BP with diameters of $130 \mathrm{~km}$ would already yield to a flux gap of two orders of magnitude. The drop of BP numbers between the histogram maximum and the $90 \mathrm{~km}$ resolution limit achieved is found to depend on the low-pass filtering and is thus probably virtual. Higher spatial resolution data will still increase the flux gap between bright and dark solar magnetic flux concentrations which might be a signature of differently deep rooting in the solar atmosphere.

Key words. Sun: activity - magnetic fields

\section{Introduction}

Small-scale magnetic flux concentrations play a dominant role among solar structures - even outside active regions and during the minimum of the solar cycle. A precise investigation of them would require Zeeman polarimetry (Stokes-V maps, e.g., Domínguez Cerdeña et al. 2003) which, however, does not yet reach sufficiently high spatial resolution below $100 \mathrm{~km}$. Fortunately, the spectral lines of the $\mathrm{CH}$ molecule near $430 \mathrm{~nm}$ (the "G-band") offer a unique possibility: magnetic structures appear as "G-band bright points" (BP) in filtergrams (cf., Muller \& Roudier 1984; Title \& Berger 1996). The observation of these BP through an interference filter of few nm width allows to take a large number of short-exposure images for, e.g., post-facto speckle reconstruction yielding images at a spatial resolution near the telescope's diffraction limit (Fig. 1).

Earlier investigations of the size distribution of the BP using the $45 \mathrm{~cm}$ Dutch Open Telescope (DOT) on La Palma (Bovelet \& Wiehr 2003) resulted in a histogram with a nearly exponential increase in the size distribution towards smaller scales, down to $220 \mathrm{~km}\left(0.3^{\prime \prime}\right)$. Using the new 1-m Swedish Solar Telescope (SST) on La Palma, Wiehr et al. (2004) could extend the size histogram down to $100 \mathrm{~km}$ diameter. That histogram showed a decrease of the number of bright points smaller than $130 \mathrm{~km}$ and larger than $250 \mathrm{~km}$, with a most frequent bright point size of $160 \mathrm{~km}$ diameter. Larger structures found earlier with smaller telescopes have evidently been decomposed into smaller ones by the unprecedented spatial resolution of the 1-m SST.
Those results needed to be confirmed, since that study was based on a rather small sample of images used for the reconstruction procedure (cf., Weigelt 1977; Pehlemann \& von der Lühe 1989; de Boer \& Kneer 1992; de Boer 1996). A proper determination of noise, important for the reconstruction, was problematic since single-exposed flat field images were not available and the noise had to be estimated directly from the data. This could have affected uncertainties at highest spatial frequencies, which these authors removed by post-facto lowpass filtering. Their histogram does therefore not reveal structures smaller than $100 \mathrm{~km}$, although the diffraction limit of SST corresponds to $70 \mathrm{~km}$ on the Sun (following the Rayleigh criterion $1.0 \times \lambda / D$ ). Besides, the seeing conditions during those observations did not permit a permanent operation of the adaptive optics. As a consequence, it is not clear whether the number decrease of BP toward small sizes might arise from those restrictions.

\section{Observations and data reduction}

In order to verify these results, the active region NOAA-0636 was observed at $\theta=21^{\circ}$ on June 21, 2004, with the Swedish 1-m Solar Telescope at La Palma (SST; Scharmer et al. 2003) using a similar set-up as done by Wiehr et al. (2004). Imaging through a $430 \pm 1 \mathrm{~nm}$ (G-band) filter gave more than $1000 \mathrm{G}$-band bright points ("BP") around a small sunspot (Fig. 1). The excellent seeing conditions assured a permanent closing of the AO circuit. Bursts of 100 images, each one with $10 \mathrm{~ms}$ exposure have been taken; the read-out of the Kodak 


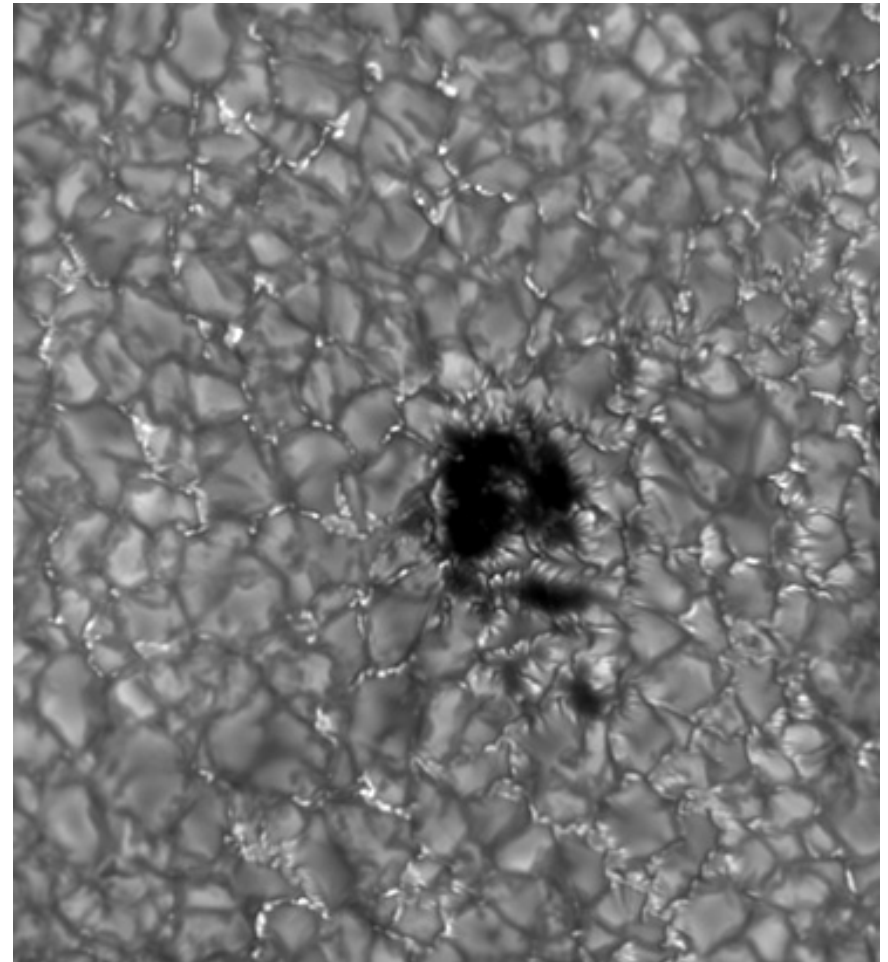

Fig. 1. Part of the speckle reconstructed G-band image used for this study, showing the active region NOAA0 10636 at $20^{\circ} \mathrm{E}, 6^{\circ} \mathrm{S}$ taken on June, 21, 2004, at the SST on La Palma; image size $30^{\prime \prime} \times 33^{\prime \prime}$; limb toward the right side.

Megaplus $1.6 \mathrm{k}$ CCD $(1536 \times 1032$ pixels $)$ was fast enough to assure a total time span for one burst of $30 \mathrm{~s}$. This corresponds, for a maximum horizontal velocity of $2 \mathrm{~km} \mathrm{~s}^{-1}$, to a lateral motion of $60 \mathrm{~km}$, and it is short as compared to typical time variations of BP (see, e.g., Bovelet \& Wiehr 2003). The pixel size

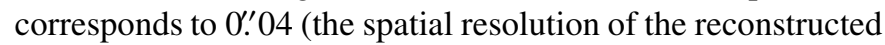
images is approximately 0 .' 12 ; see below). Flat-fields were obtained from bursts of 100 images each, taken while moving the telescope such that solar structures disappear in the average image. For a realistic determination of the noise, a separate burst of 100 single-exposed images was taken, the telescope being totally de-focused.

For the image restoration the "Göttingen speckle reconstruction code" (de Boer 1996) has been improved considering the angular dependence of the O-compensation and realistic speckle transfer functions (STF) which account for the influence of the AO (cf., Puschmann \& Sailer 2005). The speckle code recovers the Fourier phases of the "real object" using the speckle masking method (Weigelt 1977), and the Fourier amplitudes of the reconstructed image are taken from Labeyrieand spectral ratio methods (Labeyrie 1970; von der Lühe 1984). The latter determine the specific model-STF that optimally describes the atmospheric properties during the observation.

The conventional speckle reconstruction method considers only isotropic and purely atmospheric influences, and the spectral ratio $\epsilon$ is determined as the average over all iso-planar sub-fields of the image to reduce noise and uncertainties for larger wave numbers. For AO-supported observations the use of field-dependent STFs is mandatory, since the AO correction
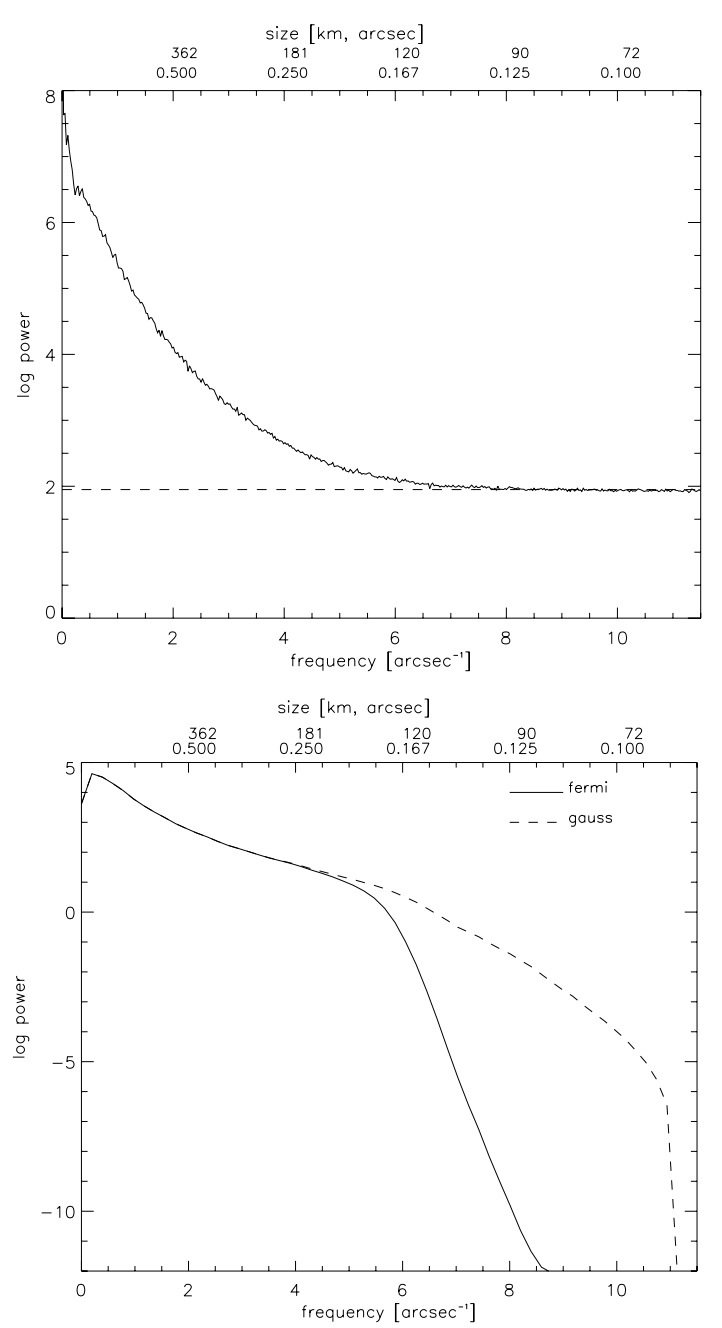

Fig. 2. Radially averaged power spectrum of the image under study (cf., Fig. 1); upper panel: raw image with reasonable noise level; lower panel: mean power of reconstructed sub-images with different filtering before their composition.

is largest near the AO lock-point and decreases towards the border of the field of view, hence, also $\epsilon$ changes with the angular distance from the lock-point. Furthermore, since the AO-compensation affects the statistics of the wave-front errors, it also influences the instantaneous $S T F$ s which are calculated for each spatial frequency via auto-correlation of the wave fronts at the telescope's pupil. As a consequence, also the shape of the STF shows the influence of the AO correction and has thus to be accordingly modelled for a correct intensity amplification of all image parts to be restored. A realistic intensity amplification through the whole image is important, since the detection algorithm essentially depends on the intensities of the features to be detected.

Even such a restoration of images obtained under permanent operation of the $\mathrm{AO}$ does not guarantee a final spatial resolution reaching the instrumental limit of the $1 \mathrm{~m} \mathrm{SST}$. Our raw best-image has a power spectrum which reaches a constant level at $u \approx 8$ ( $0 . ' 125$; resp. $90 \mathrm{~km}$; cf. Fig. 2, upper panel). In order to avoid an amplification of this noise beyond $u=8$ in the reconstruction, we applied different low-pass filters. The optimum filter used by de Boer (1996) was replaced by 
alternately a Fermi and a Gaussian filter in order to cut the high spatial frequencies as steep as possible. The Fermi filter smoothly reaches zero transmission at the $u \approx 8$ limit in our raw data. The Gaussian filter steeply cuts at the theoretical SST limit $u=11$, thus allowing the reconstruction code to consider additionally high spatial frequencies $8<u<11$. The power spectra of the reconstructed images correspondingly show for the steep Gaussian filter a marked decrease at $u \approx 10.5$, for the flatter Fermi filter, however, at $u \approx 6$ (Fig. 2).

\section{Pattern recognition}

The G-band bright points (BP) were recognised by means of the "Multi-Level Tracking" algorithm by Bovelet \& Wiehr (2001). For the lower detection threshold of the MLT algorithm we took the mean photospheric intensity in order to account for the brightness excess defining the G-band "bright" points. Among the two families of BP (Langhans et al. 2002), the magnetic ones were selected by an additional local contrast criterion which accounts for their location in intergranular lanes (Bovelet \& Wiehr 2003).

Since the active region was not observed exactly at disk centre, a number of BP show a "projection" on the limb-wards neighbouring granule, indicating the appearance of "limb facular grains" at rather small helio-centric angles near $21^{\circ}$ (see also Hirzberger \& Wiehr 2005). In order to avoid any influence of this "projection effect" on the size histogram, the final sample has been reduced to 950 largely circular BP.

The MLT algorithm returns several parameters (cf., Bovelet $\&$ Wiehr 2003), among which the size of each feature detected is given in pixel-areas. We set the lower size limit to $4 \mathrm{px}$ (e.g., 2 px $\times 2$ px, i.e. $\left.0.081^{\prime \prime} \times 0.081^{\prime \prime}\right)$. This corresponds for June 21 to $60 \mathrm{~km} \times 60 \mathrm{~km}$ being just below the Rayleigh limit of the SST which amounts to $70 \mathrm{~km}$ for $430 \mathrm{~nm}$. We assign each feature the diameter of a circle covering the same area as determined by the pattern recognition algorithm.

\section{Size distribution of inter-granular magnetic features}

The diameter histograms in Fig. 3 show different distributions for the two low-pass filters: the higher spatial frequencies passing the Gaussian filter significantly yield more BP with diameters between 90 and $150 \mathrm{~km}$. The steep drop of the BP numbers at $90 \mathrm{~km}$ (Fig. 3) for the Gaussian filter well corresponds to the limiting value $u=8$ (Fig. 2; upper panel) and establishes that no realistic features are recognised beyond that point.

The decrease of BP numbers with diameters larger than $180 \mathrm{~km}$ is found to originate almost entirely from a segmentation of closely neighbouring BP occurring as "chains" (resp. filigree "crinkles"; Dunn \& Zirker 1973). Evidently, the flatter decrease of the power toward larger frequencies for the Gauss filtering yields a better segmentation of closely neighbouring BP. This affects most of the increase of BP numbers with diameters below $150 \mathrm{~km}$.

We artificially segmented all elongated BP with corresponding "round" areas of diameters larger than $120 \mathrm{~km}$. The

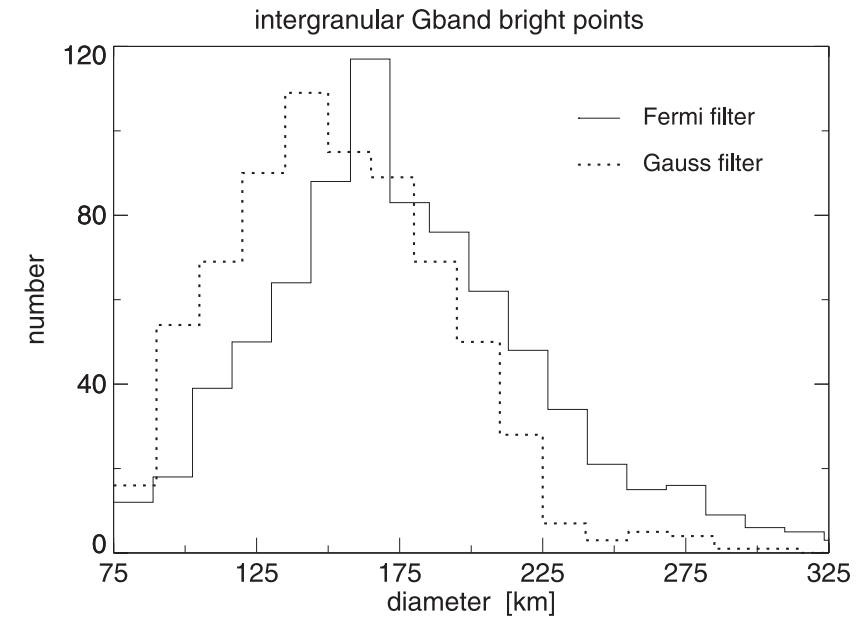

Fig. 3. Diameter histogram for 954 intergranular G-band bright points for the two low-pass filters with different cut-off affecting the power spectra shown in Fig. 2.

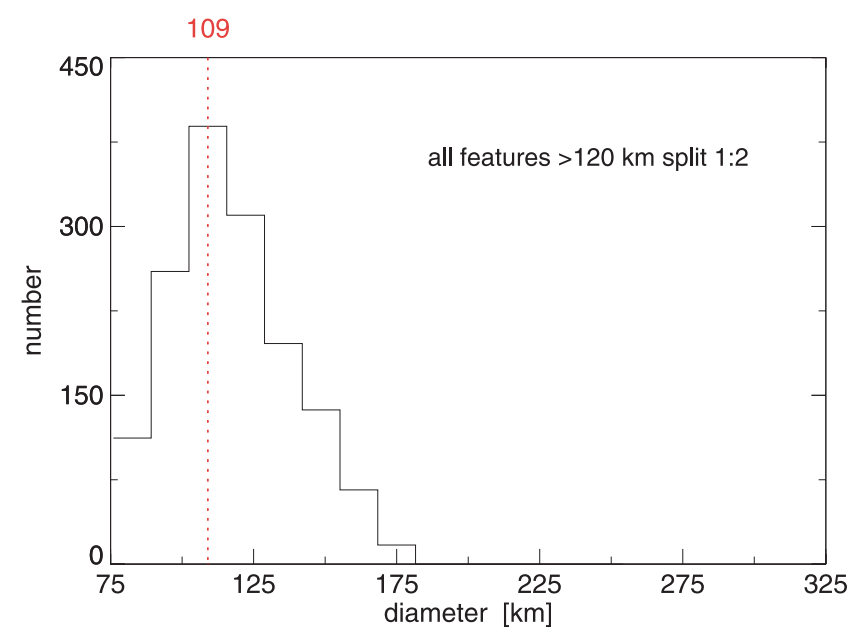

Fig. 4. Diameter histogram for the intergranular G-band bright points detected after low-pass filtering with the Gauss filter with artificial segmentation of elongated structures.

resulting histogram in Fig. 4, indeed, shows a decrease at diameters between $175 \mathrm{~km}$ and $225 \mathrm{~km}$. As expected, the "tail" from Fig. 3 has completely disappeared.

\section{Conclusions}

Our results establish the former finding of a "gap" between small-scale magnetic features, appearing bright in the G-band, and smallest dark pores. At high spatial resolution achieved under permanent operation of the adaptive optics at the $1 \mathrm{~m} \mathrm{SST}$, we find (with the steep filter) only few BP of more than $225 \mathrm{~km}$ equivalent "round" diameter. This corresponds to a total magnetic flux of maximally $3.1 \times 10^{17} \mathrm{Mx}$, assuming the most frequent flux density of $785 \mathrm{Mx} / \mathrm{cm}^{2}$ found by Berger et al. (2004). This value is more than 30 times smaller than that for smallest pores of $1000 \mathrm{~km}$ diameter and $2000 \mathrm{Gs}$ which, in turn, correspond to the lower flux limit of $10^{19} \mathrm{Mx}$ given by the kink instability (Meyer et al. 1977). Further (artificial) segmentation simulating still higher spatial resolution yields an upper flux 
limit below $2 \times 10^{17} \mathrm{Mx}$. A flux "gap" of two orders of magnitude would occur for BP dimeters of $130 \mathrm{~km}$.

Still smaller BP might even increase this flux difference between bright and dark organised solar magnetic flux concentrations. It may be explained by a differently deep rooting in the solar atmosphere. Bright points are known to be markedly affected by granular motions (e.g. Bovelet $\&$ Wiehr 2003) which is not the case for (even smallest) pores. A possible explanation is suggested by the fact that pores are members of active regions and thus probably deeper rooted than BP. If the flux gap were filled by magnetic knots, which are reported to be neither visible in the continuum (Beckers \& Schöter ) nor in the G-band (Berger et al. 2004), it would be of interest to study their behaviour within the granular motions from Stokes-V maps.

Acknowledgements. We thank B. Bovelet for performing the histogram with his MLT algorithm. K.G.P. thanks the Deutsche Forschungsgemeinschaft DFG for support through grant KN 152/29-1. The data were obtained during a campaign supported by the the European Union (OPTICON Trans-National Access Program) together with M. Sobotka. The SST is operated by the Swedish Academy of Sciences at the Spanish Observatorio del Roque de los Muchachos (IAC).

\section{References}

Beckers, J. M., \& Schröter, E. H. 1968, Sol. Phys., 4, 165

Berger, T.E., Rouppe van der Voort, L. H. M., Löfdahl, M. G., et al. 2004, A\&A, 428, 613

Bovelet, B., \& Wiehr, E. 2001, Sol. Phys., 201, 13

Bovelet, B., \& Wiehr, E. 2003, A\&A, 412, 249

de Boer, C. R. 1996, A\&AS, 120, 195

de Boer, C. R., \& Kneer, F. 1992, A\&A, 264, L24

Domínguez Cerdeña, I., Sánchez Almeida, J., \& Kneer, F. 2003, A\&A, 407,741

Dunn, R. B., \& Zirker, J. B. 1973, Sol. Phys., 33, 281

Hirzberger, J., \& Wiehr, E. 2005, A\&A, 438, 1059

Labeyrie, A. 1970, A\&A, 6, 85

Langhans, K., Schmidt, W., \& Tritschler, A. 2002, A\&A, 394, 1069

Meyer, F., Schmidt, H. U., \& Weiss, N. O. 1977, MNRAS, 179, 420

Muller, R., \& Roudier, T. 1984, Sol. Phys., 94, 33

Pehlemann, E., \& von der Lühe, O. 1989, A\&A, 216, 337

Puschmann, K. G., \& Sailer, M. 2005, A\&A, submitted

Scharmer, G. B., Bjelksjo, K., Korhonen, T. K., Lindberg, B., \& Pettersen, B. 2003, Proc. SPIE, 4853, 370

Title, A. M., \& Berger, T. E. 1996, ApJ, 463, 797

von der Lühe, O. 1984, J. Opt. Soc. Am., A1, 510

Weigelt, G. P. 1977, Optics Comm., 21, 55

Wiehr, E., Bovelet, B., \& Hirzberger, J. 2004, A\&A, 422, L63 